Bateman, in all probability, never saw such a case, or they also might have made a species of it. The result of the removal of this large mass of mortar-like granules, and the destruction of vermin, was, with the addition of a measure to which $I$ shall hereafter more particularly allude, a perfect cure. The subject of this case was an idle vixen, who had refused attention or applications of any sort for a long time. She had inoculated other children in the earlier period of the disorder, but they having submitted to due remedial meaus, never exhibited any of the features of the mortar disease.

At about the same period at which the case I have detailed came under my observation, two children were brought to me from the neighbourhood of DruryLane, the one with extensive ring-worm as we commonly see it, the other with a cluster of lupine-seed scabs. I found that the first, which was evident from its appearance, had been kept in a moderate state of cleanliness; the other was emaciated and covered with filth, and had had no application whatever made to the head. Many other cases have since occurred to justify me in declaring that the circular ring-worm, or p. scutulata and p. lupinosa, are the same disease, and that p. furfurans, or t. furfuracée, or amiantacée, are merely its sequelæ under bad management.

The simple circular contagious ringworm is not, as has been supposed by many, produced only by infection or contagion. It arises in a very large portion of cases from the same sources as other diseases of the skin, such as improper diet, producing constipation of the bowels; restraint of the due and healthy exercise of children; repletion from over feeding, or from merely a single indulgence of sweet-meats or cakes, producing acidity. Yet thus originating it is quite as contagious as that which has spread directly in a family, from child to child, by contact, where no derangement of the stomach or system can be traced or suspected. Among children with a light complexion, their slsin being, for the most part, more irritable, the disease produces in them inore itching and discomfort than under other circumstances and spreads more rapidly; the hair, also, on account of its more delicate formation, yields to its destructive influence at once, so that, occasionally, in the course of a single night, you may pull out, without the child being aware of it, hundreds of hairs which the day before appeared to have a firm hold in the scalp. The extreme fineness of the hair, too, and it is, generally, proportionately thickly distributed, prevents the formation of even a minute vesicle, and a continual oozing of the contagious fluid goes on, producing an abundant supply of the latter. Hence the disease spreads far more rapidly in families of a light complexion and delicacy of skin and hair, than in others of an opposite description.

The history and causes of this disease being thus briefly disposed of, I shall proceed to show in my next its modus ope. randi in producing its distinguishing fea. ture on the scalp: the manner in which it acts in the exercise of its manifestly withering influence on the hair of the latter. If you apply a blister over the whole scalp, you find that, violent as is the irritation, great as is the discharge, the hair grows as healthy as before, after its influence has ceased, while a tiny disease like ring-worm may exist for yearshardly traceable by any sign but a destruction of the hair, the most disfiguring and vexatious; and yet, looking at it in a constitutional point of view, the most insignificant of all human disorders.

\section{CORRESPONDENCE WITH THE}

\section{APOTHECARIES' COMPANT}

REIATIVE TO THA PROSECUTION OF AN

UNLICENSED PRACTITIONER.

\section{To the Editor of The Lancex.}

Sir,-In The Lancet of February $26 \mathrm{th}$, No. 600, there is a letter from "A Country Practitioner," together with one from Mr. Upton, clerk to the Apothecaries' Company, respecting the Act of 1815. In consequence of having read the same 1 take the liberty of sending you the following, and I think that those who are interested in the subject will conclude with the correspondent alluded to, in asking " Of what use is the Act of 1815 as a protection to the general practitioner ?"

I am, Sir, your obedient servant, John S. Hallows.

62, Brownlow-hill, Liverpool, March 9th, 1835.

"To the Chairman and Court of Examinens, Apothecaries' Hall, London.

"Gentumen, - Having obtained my. certificate to practise as an apothecary in 1822, I consider it my duty, as a general practitioner, to transmit to you the following statement, presuming you will (with me and most of the profession here) be of opinion that the individual alluded to is a very unfit person to practise as an apothecary, and is one against whom a 
prosecution ought to be instituted according to the provisions of the Act.

"A bout nine years ago Francis Edward O'Gorman made application to Mr. Andrew Gillon, a general practitioner in this town, to become an apprentice, and as he could not afford to give a premium, his proposal was to serve a longer apprenticeship than usual, and to make himself generally useful in cleaning boots, shoes, knives, forks, harness, take care of the gig, \&c., as an equivalent; but Mr. Gillon declined the proposal, and he soon afterwards entered into the employ of a druggist in Marybone, in this town, of the name of Bainbridge. Whilst with him he was pretty attentive to the shop, and was generally respected by $\mathrm{Mr}$. Bainbridge's customers. Finding himself in this confidential situation, he wrote to Ireland for his brother, John O'Gorman, a druggist, who came over and opened a shop one or two doors from Mr. Bainbridge's, and Francis Edward O'Gorman, became an apprentice or shopman to him, much to the injury of Mr. Bainbridge, who was in consequence obliged to give up business, and the whole of his customers became dealers with the O'Gormans. When this youth had been in the shop of his brother about three years, he commenced the profession of an apothecary in Brownlow-hill, in this town, placing on his front door, 'O'Gorman, Surgeon,' at the same time keeping an open apothecary's shop, going out regularly to visit patients, prescribing and sending out medicines made up by him, or under his superintendence, and he has thus continued practising as an apothecary for about five years, much to the annoyance of the profession who have been at the expense, trouble, and anxiety of obtaining certificates from the Court of Examiners as by law establishel. The whole of what I now communicate $I$ am in a situation to prove, and also that he is practising contrary to the provisions of the Act, in confirmation of which $I$ send you a copy of one of his bills delivered to a patient of his whom he was attending and treating for dyspepsia, when in reality the case was hepatitis; this can also be proved by a Mr. Barrow, a general practitioner in Gloucester-st., in this town, who was called to visit this patient, and who attended her during the remainder of her sickness, she not having been benefited by the attendance, \&c., of Francis Edward O'Gorman. You will perceire, at the bottom of the bill, ' 45 , Great Cross Hall-street, and 53, BrounlowHill;' the former street being the now residence of John O'Gorman, the latter the residence of Francis Edward O'Gor-, man, the indiridual in question. The labels on the bottles in which he sends out medicines have the two addresses printed upon them, which no doubt is done intentionally, with a view to evade the Act in the event of a prosecution, by saying one is an assistant to the other. I must here observe, that all the drugs sent to the shop of Francis Edward O'Gorman are invoiced in his own name, but when any one disputes the amount of his bill, or will not pay at all, then he sues in the Liverpool Court of Requests, and invariably recovers in the name of his brother. I understand from those connected with this Court, that within the last few years he has recovered upwards of $300 l$., all for medical attendance, \&c., in opposition to the Act.

"I am not sending this statement from any personal ill feeling towards Mr. Francis Edward O'Gorman, hut on public grounds, as I consider he is not possessed of sufficient medical ability to be intrusted with cases affecting the lives of individuals, and that his continuing to practise as an apothecary, is also not only a loss to the regular practitioner, but a direct invasion on the rights and privileges of the "Worshipful the Apothecaries" Company.' Hoping I may soon have a reply to what I have here written, 1 am, Gentlemen, with the greatest respect, your obedient servant, "Johy S. Hallows. “62, Brownlow-hill, Liverpool, June 16th, 3834."

"Copy of Bill in my possession.

" ' Mr. Hollands to J. O'Gorman, Surgeon, Dr.

“ " To medicines for Mrs. H. . £ $£ \quad 5 \quad 2$ Bleeding and visits $\ldots \ldots \ldots, 0 \quad 0 \quad 2 \quad 6$

“ 45, Great Cross Hall-street, 53 , Brownlow Hill, May 1834." "Addressed to Mr. Hollands."

[The above letter was directed to the Chairman and Court of Examiners, in consequence of there not being at the time a clerk to the Company.-J.S.H.]

\section{Mr. Upton's Reply to my Letter.}

"Apothecaries' Hall, 30th June, 1834. " Sir,-Your letter of the 16th instant, addressed to the Chairman and Court of Examiners has been placed in my hands, and its not having received an earlier notice, is owing to a vacancy in the oftice of clerk to this Society, which has only recently been filled up. Before proceedings 